\title{
The utility of telemedicine in pediatric emergency care: A scoping review.
}

\section{Owen Robinson, Shaelynn Zouboules, Hailey Lafave, Roger Galbraith, Eddy Lang}

Background: Widespread public health measures to combat COVID-19 and escalated parental fear in seeking medical care have compromised access to acute healthcare, leading clinicians to search for alternative methods of delivery. Pediatric emergency departments (ED) have seen significant reductions in documented visits without evidence of a reduction in needs. In Alberta, average daily visits to pediatric emergency and urgent care departments decreased $69.6 \%$, from 952.2 in December 2019 to 289.6 in April 2020. While pediatric emergency telemedicine (PET) programs have the potential to alleviate said gaps in care, it is critical that these technologies are evaluated to ensure patient safety and efficacy.

Implementation: This study aimed to serve as an implementation framework for future PET programs. A scoping review was conducted in accordance with the preferred reporting items for systematic reviews and meta-analysis extension for scoping reviews (PRISMA-ScR). The primary objective was to map the existing literature and identify research gaps pertaining to the use of telemedicine in pediatric emergency departments. Primary areas of focus included directto-consumer (DTC) telemedicine, rural/regional applications, general ED utility, transfer of care and specialist consultation. This presentation focused on the aspects of DTC telemedicine, and its ability to potentially alleviate the present barriers to in-person presentations to EDs for acute pediatric health concerns. Our team consisted of two University of Calgary affiliated emergency physicians, three University of Calgary medical students, Canadian Agency for Drugs and Technology in Health research consultants, and a university librarian.

Evaluation Methods: The outcomes of interest that we used to evaluate the relevant literature included: prevalence of PET; current applications; patient outcomes; patient satisfaction; provider satisfaction; and feasibility, challenges and barriers to implementation. In addition, we aimed to determine the proportion of literature focusing on DTC telemedicine, as this would be the desirable telemedicine application that could be used to supplement the gap in pediatric ED visits during the pandemic and mitigate the resulting health impacts. Lastly, we aimed to characterize both successes and challenges associated with DTC telemedicine in order to provide guidance for future research and policy.

Results: Searches of the electronic databases returned 1617 studies. Following the two-step screening process, 37 studies met our inclusion criteria and six focused on DTC telemedicine. Study designs were all observational with all published in 2015 or later. The number of studies reporting data on the outcomes of interest were as follows: patient satisfaction $(\mathrm{N}=0)$; prevalence $(\mathrm{N}=1)$; provider satisfaction $(\mathrm{N}=1)$; patient outcomes $(\mathrm{N}=2)$; current applications $(\mathrm{N}=6)$; feasibility, challenges and barriers $(\mathrm{N}=6)$. Respiratory presentations were the most prevalent application. Three of six studies demonstrated agreement between telemedicine and in-person providers during acute assessments, demonstrating reliability of telemedicine. Conversely, two 
studies conveyed antimicrobial stewardship with conflicting results. Overall, results were largely positive with important challenges identified.

Advice and Lessons Learned: Based on the lessons learned from our research, we recommend the following:

1) Implentation of a DTC telemedicine program can provide timely access to care, while minimizing the health risks associated with visting the ED during the COVID-19 pandemic.

2) Respiratory complaints were among the most common presentations and thus we recommend developing diagnostic and management algorithms to standardize the virtual care provided.

3) Continue quality improvement measures upon implementing a telemedicine program through timely feedback regarding physicians' experiences and challenges in order to address concerns early and optimize efficacy of the program. 